\title{
Evaluation of xerostomia closely associated with systemic diseases using a dental approach
}

\author{
Hiroshi Mori $^{\mathbf{1}^{* \#}}$, Noriyuki Hoshi ${ }^{2 *}$, Motoe Taniguchi ${ }^{1}$, Masako Banka ${ }^{2}$, Katsuhiko Kimoto ${ }^{2}$ \\ ${ }^{1}$ Maxillofacial Diagnostic Science, Department of Oral Diagnosis, Kanagawa Dental College, Yokosuka, Japan \\ ${ }^{2}$ Division of Fixed Prosthodontics, Department of Oral and Maxillofacial Rehabilitation, Kanagawa Dental College, Yokosuka, Japan \\ Email: " morihiro@kdcnet.ac.jp
}

Received 28 July 2012; revised 30 August 2012; accepted 7 September 2012

\begin{abstract}
Based on the findings of epidemiological surveys in western countries, the number of person with suspected xerostomia in Japan is estimated to be $30,000,000$. Xerostomia is caused by: 1) Systemic diseases; 2) Medications; 3) Tumors or trauma; 4) Radiotherapy; and 5) Neurological factors. Its symptoms can be alleviated, and its progression can be stopped by close cooperation between the dental and medical departments. However, at present, symptomatic treatment methods such as water drinking, oral rinsing with water, and administration of Kampo medicines or parasymptomimetic drugs are mainly used, and a standard treatment has not been established. On the other hand, previous studies on xerostomia have reported improvement in dry mouth symptoms using dental approaches or a relationship between the development of candidiasis and the salivary flow rate. Therefore, in this study, to establish a dental method useful for improving xerostomia associated with systemic diseases and medications for them, we restored occlusal function, and as a result, increased the salivary flow rate, and evaluated changes in oral findings.
\end{abstract}

Keywords: Xerostomia; Saliva; Prosthetic Treatment

\section{INTRODUCTION}

Based on the findings of epidemiological surveys in western countries, the number of person with suspected xerostomia in Japan is estimated to be $30,000,000$ [1]. However, many persons have not visited hospitals, and the diagnostic and treatment methods for xerostomia have not been established because the patients may visit various departments depending on the underlying disorder.

The symptoms and signs of xerostomia include dry

${ }^{*}$ Equally contributed authors.

${ }^{\#}$ Corresponding author. mouth, oral pain, oral discomfort, dysgeusia, dysphagia, a decrease in the oral $\mathrm{pH}$, an increase in dental caries, and fungal infection as a complication, which markedly aggravate the oral environment, reducing patients' quality of life (QOL). Xerostomia is caused by: 1) Systemic diseases; 2) Medications; 3) Tumors and trauma (their sequelae); 4) Radiotherapy; and 5) Neurological factors.

Concerning causes 1) and 2), systemic diseases tend to become chronic, prolonging the drug administration period, which results in aggravation of the symptoms of xerostomia. This aggravation increases dental caries and worsens periodontal disease. Due to loss of teeth and dislodgement of restorations and prostheses, masticatory function decreases, and the oral hygiene status becomes poor. Malnutrition results from a decrease in eating function, and candidiasis develops due to the poor oral environment. Candida induces aspiration pneumonia. Thus, close cooperation between the dental and medical departments is indispensable.

In addition, the symptoms of xerostomia can be alleviated, and its progression can be stopped by appropriate treatment in the dental and medical departments.

At present, the treatment methods include water drinking, oral rinsing with water, use of artificial saliva, and use of oral moisturizers [1]. For xerostomia due to Sjögren syndrome, symptomatic treatment with anetholtrithione as a cholagogue, bromhexine hydrochloride and ambroxol hydrochloride as expectorants, Kampo medicines such as bakumon-do-to, or pilocarpine as a parasymptomimetic drug is mainly performed, and a standard treatment have not been established.

Previous studies on xerostomia have reported improvement in dry mouth symptoms using dental approaches or a relationship between the development of candidiasis and the salivary flow rate [2-4].

Therefore, in this study, to establish a dental method useful for improving xerostomia due to systemic diseases and associated medications, we restored occlusal function, and as a result, increased the salivary flow rate, and evaluated changes in oral findings. 


\section{SUBJECTS AND METHODS}

\subsection{Subjects}

The subjects consisted of 40 patients ( 15 males and 25 females) aged 43 - 83 years (mean, 63.85 years) who visited the hospital affiliated with our university between 2000 to 2007, required occlusal treatment, and also complained of oral dryness and discomfort.

The patients were classified into two groups: those with underlying diseases under treatment (Group 2) or those without underlying diseases (Group 1). Group 1 consisted of 20 patients ( 8 males and 12 females) aged 48 - 83 years (mean, 63.85 years), and Group 2 consisted of 20 patients ( 7 males and 13 females) aged $43-76$ years (men, 59.65 years).

\subsection{Tooth Missing Status at the Initial Consultation}

The tooth missing pattern at the time of the initial consultation was classified according to the Eichner index.

\subsection{Measurement of the Salivary Flow Rate}

The resting and stimulated salivary flow rates were measured using the saliva spitting method. For the measurement of the resting salivary flow rate, saliva was collected for 15 minutes in a paper cup, and its volume was measured. Decreased salivary flow rates were defined as $\leq 1.5 \mathrm{ml} / 15$ minutes.

The stimulated salivary flow rate was measured using the gum test. During gum (Lotte Free Zone/Tokyo) chewing, whole saliva was collected for 10 minutes in a paper cup, and its volume was measured. Decreased salivary flow rates were defined as $\leq 10 \mathrm{ml} / 10$ minutes. Measurements were performed in a quiet situation at about 10:00 in the morning more than 1 hour after breakfast.

\subsection{Oral Findings}

The tongue (redness, erosion, smoothness, fur), palate (redness, erosion), buccal mucosa (redness, erosion), mouth angles (cheilosis angularis), and the oral cavity (aphtha, ulcer) were observed. The relationships between changes in oral findings and those in the salivary flow rate as well as the oral hygiene status were evaluated.

\subsection{Study Period}

At the time of the initial consultation, in addition to oral hygiene instruction and prosthetic treatment, the salivary flow rate was measured, and the oral cavity was examined. After 1 - 2 weeks, at the time of prosthesis fitting, and after 6 months, 1 year, and 3 years, measurement of the salivary flow rate and examination of the oral cavity were performed.

\subsection{Statistical Analysis}

Differences were analyzed using repeated 2-way ANOVA, and $p<0.05$ was regarded as significant.

\section{RESULTS}

\subsection{Status of Treatment for Underlying Diseases}

In Group 2, diabetes mellitus was observed in 10 patients (that complicated by other diseases in 6), hypertension in 7 (that complicated by other diseases in 3), Sjögren syndrome in 4 (that complicated by other diseases in 3), and other diseases under treatment in 7 (Table 1).

All patients, excluding 1with diabetes mellitus receiving only dietary therapy and 1 with Sjögren syndrome using only a mouthwash, were receiving drugs.

\subsection{Tooth Missing Status at the Initial Consultation and Contents of Prosthetic Treatment}

In Group 2, Eichner Class A was observed in 4 patients, Class B in 8, and Class C in 8. In Group 1, Class A was observed in 7 patients, Class B in 10, and Class C in 3 (Table 2). The tooth missing status according to the Eichner index was slightly be severer in Group 2.

Concerning the prosthetic treatment, new denture fabrication was performed in 11 patients, denture and occlusal adjustments in 7, and only hygiene instruction without denture problems in 2 in Group 1. In Group 2, new denture fabrication was performed in 15 patients and denture and occlusal adjustments in 5; no patient received only oral hygiene instruction without denture problems (Table 3).

Table 1. Underlying disorders.

\begin{tabular}{cc}
\hline Underlying disorders & Patients \\
\hline Diabetes mellitus & 7 \\
Hypertension & 6 \\
Sjögren syndrome & 4 \\
Depression & 1 \\
Angina pectoris & 2 \\
Osteoporosis & 2 \\
Myocardial infarction & 1 \\
Cerebrl infarction & 1 \\
\hline
\end{tabular}

Table 2. Eichner index.

\begin{tabular}{ccc}
\hline & Group 1 & Group 2 \\
\hline Class A & 7 & 4 \\
Class B & 10 & 8 \\
Class C & 3 & 8 \\
\hline
\end{tabular}

Table 3. Treatment contents.

\begin{tabular}{ccc}
\hline & Group 1 & Group 2 \\
\hline New denture fabrication & 11 & 15 \\
Denture adjustment-Occlusal & 7 & 5 \\
Hygiene instruction & 2 & 0 \\
\hline
\end{tabular}




\subsection{Salivary Flow Rate}

In Group 1, the resting salivary flow rate at the initial consultation was $2.38 \pm 0.70 \mathrm{ml} / 15 \mathrm{~min}$, which exceeded the lower limit of the reference value $(1.5 \mathrm{ml} / 15 \mathrm{~min})$, and $3.09 \pm 0.87 \mathrm{ml}$ after 3 years, showing a significant increase. In Group 1, there was no patient showing a resting salivary flow rate below the reference value at the initial consultation. In this group, only 1 patient showed a decrease at the time of prosthesis fitting compared with the value at the initial consultation and the same value as the initial value after 3 years, and 4 patients showed no changes after prosthesis fitting compared with the value at the initial consultation. Of the 4 patients, 2 showed an increase after 6 months, and 1 of the other 2 showed a slight decrease after 6 months, a decrease after 1 year compared with the value at the initial consultation, but a slight increase after 3 years compared with the initial value. In the other patient, no increase was observed even after 6 months compared with the value at the initial consultation (Figure 1).

In Group 2, the resting salivary flow rate at the initial consultation was $1.71 \pm 0.58 \mathrm{ml} / 15 \mathrm{~min}$, slightly exceeding the lower limit of the reference value $(\geq 1.5 \mathrm{ml} /$ $15 \mathrm{~min}$ ), and $1.95 \pm 0.65 \mathrm{ml}$, showing a slight increase after 3 years. However, the rate of increase $(14.07 \%)$ in this group was lower than that in Group 1 (29.98\%). In Group 2 , the resting salivary flow rate at the initial consultation was below the reference value in 6 patients, of whom 4 had a history of Sjögren syndrome. The value decreased at the time of prosthesis fitting in 2 patients, both of whom had Sjögren syndrome. One of them showed no changes in the flow rate at the time of prosthesis fitting compared with the value at the initial consultation but slight increases after 6 months and 1 year, and a slight decrease after 3 years. The other showed a peak value $(\geq 1.0 \mathrm{ml} / 15 \mathrm{~min})$ at the time of the initial consultation, decreases after 1 - 2 weeks, reaching the minimum value $(\geq 0.7 \mathrm{ml} / 15 \mathrm{~min})$ after 6 months, and a decrease $(\geq 0.9$

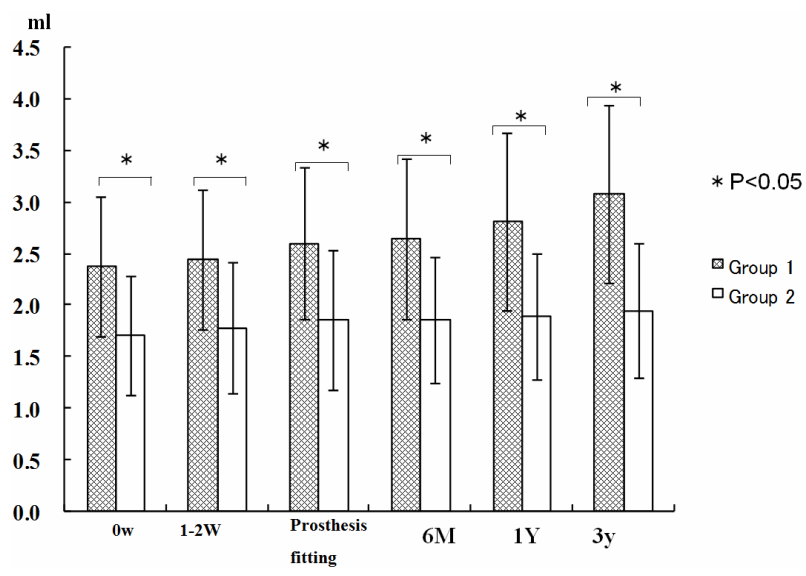

Figure 1. Resting salivary flow rate. $\mathrm{ml} / 15 \mathrm{~min}$ ) after 3 years compared with the value at the initial consultation (Figure 1).

In Group 1, the stimulated salivary flow rate at the initial consultation was $11.20 \pm 3.67 \mathrm{ml} / 15 \mathrm{~min}$, exceeding the lower limit of the reference value ( $\geq 10 \mathrm{ml} / 10 \mathrm{~min})$ and $16.26 \pm 2.46 \mathrm{ml} / 15 \mathrm{~min}$ after 3 years, showing a significant increase. In 12 patients in this group, the flow rate was below the reference value at the initial consultation, but increased at the time of prosthesis fitting, exceeding the lower limit of the reference value, and remained increased after 1 and 3 years (Figure 2).

In Group 2, the stimulated the stimulated salivary flow rate at the initial consultation was $8.66 \pm 1.95 \mathrm{ml} / 15 \mathrm{~min}$, being below the reference value $(\geq 10 \mathrm{ml} / 10 \mathrm{~min})$, but $12.56 \pm 2.96 \mathrm{ml} / 15 \mathrm{~min}$ after 3 years, exceeding the lower limit of the reference value. In this group, there were 12 patients showing a value below the reference value at the initial consultation. A decrease in the flow rate compared with the value at the initial consultation was observed at the time of prosthesis fitting in 2 patients. One of the two patients had Sjögren syndrome, and showed an increase after 6 months compared with the initial value, a decrease after 1 year, and an increase after 3 years compared with the initial value, but the value after 3 years was still $6.9 \mathrm{ml} / 10 \mathrm{~min}$, being below the reference value. One patient showing a decrease at the time of prosthesis fitting was receiving a drug due to depression. However, the values after 6 months, 1 year, and 3 years, were slightly increased, slightly exceeding the lower limit of the reference value. In 4 patients, the value at the time of prosthesis fitting increased compared with that at the initial consultation, but was below the reference value. Of the 4 patients, 3 had Sjögren syndrome but showed increases after 6 months, 1 year, and 3 years, including 1 showing a value exceeding the lower limit of the reference value after 3 years (Figure 2).

Concerning the influences of underlying diseases on the salivary flow rate in Group 2, only in the group with Sjögren syndrome, the resting salivary flow rate at the

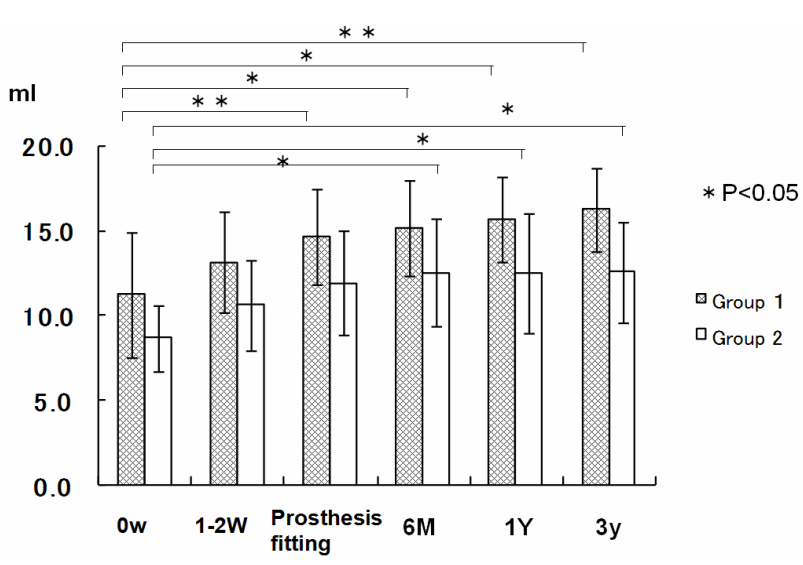

Figure 2. Stimulated salivary flow rate. 
initial consultation was below the reference value and significantly lower than that in the groups with the other diseases. The group with Sjögren syndrome also showed values above the reference value both after 6 months and 3 years. In all underlying disease groups, the value tended to gradually increase with time at the time of prosthesis fitting and after 6 months, 1 year, and 3 years (Figure 3).

The stimulated salivary flow rate was below the reference value at the initial consultation for all underlying diseases, but exceeded the reference value after 6 months for all diseases, and remained higher than the reference value after both 1 and 3 years (Figure 4).

\subsection{Oral Findings}

In Group 1, the frequent findings at the initial consultation were tongue redness $(75 \%)$, tongue erosion $(65 \%)$, tongue fur $(50 \%)$, buccal mucosal redness $(35 \%)$, and tongue smoothness $(30 \%)$. In Group 2, oral findings and their incidences decreased at the time of prosthesis fitting;

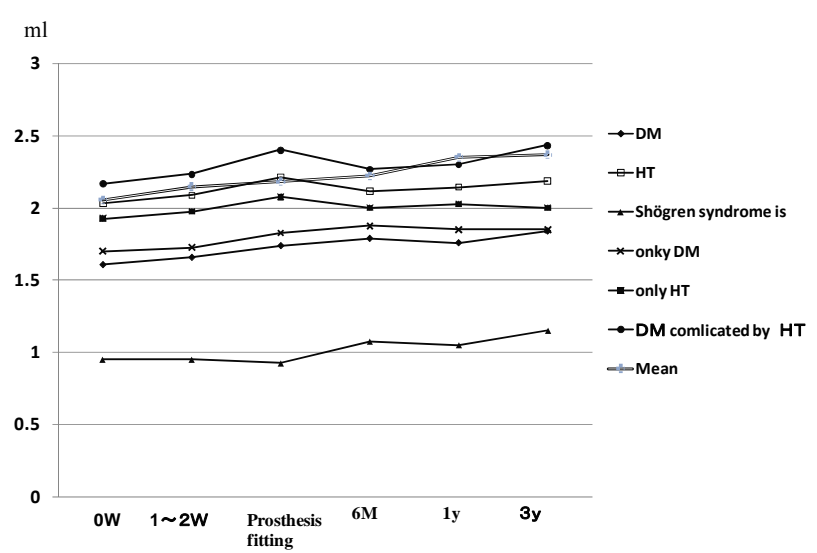

Figure 3. Resting salivary flow rate according to disorder in Group 2.

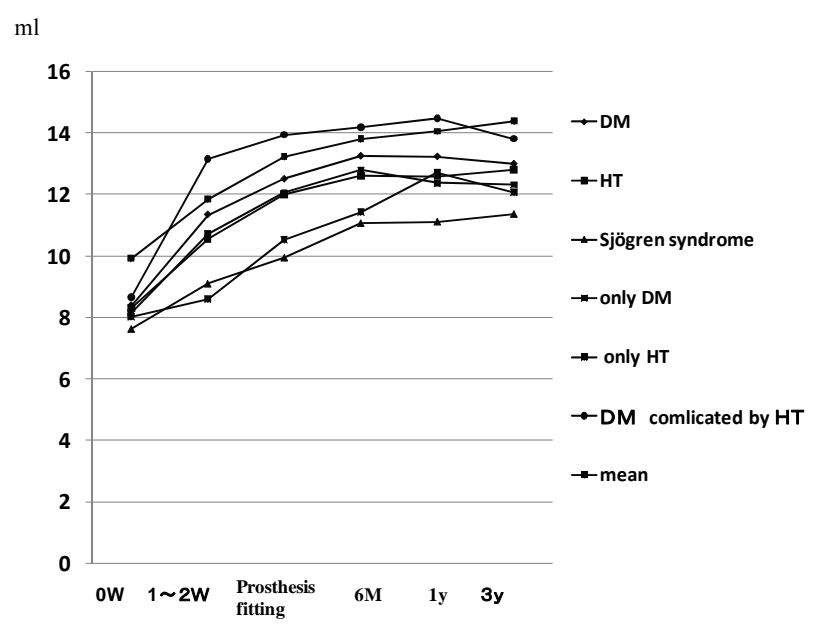

Figure 4. Stimulated salivary flow rate according to disorder in Group 2. tongue redness $(5 \%)$, tongue erosion $(5 \%)$, tongue smoothness $(5 \%)$, and tongue fur $(5 \%)$ were observed, but other findings were absent. There was no finding after 1 or 3 years (Table 4).

In Group 2, the frequent findings at the initial consultation were tongue redness $(90 \%)$, tongue erosion $(85 \%)$, tongue fur $(70 \%)$, buccal mucosal redness $(70 \%)$, and tongue smoothness $(60 \%)$. The incidences of oral findings tended to decrease at the time of prosthesis fitting and after 6 months: tongue redness $(45 \%)$, tongue erosion $(35 \%)$, tongue smoothness $(25 \%)$, and tongue fur $(25 \%)$ at the time of prosthesis fitting; tongue redness $(35 \%)$, tongue smoothness $(20 \%)$, and palatal erosion $(20 \%)$ after 6 months. However, oral findings were still present even after 1 and 3 years: tongue redness $(30 \%)$ and tongue smoothness (20\%) after 1 year; tongue redness $(25 \%)$ and tongue smoothness $(20 \%)$ after 3 years. Thus, oral findings did not completely disappear in Group 1 (Table 5).

\section{DISCUSSION}

The maximum incidence of xerostomia was reported to be $25 \%$ of the population [5], and the estimated number of people in Japan with this disease is about 30,000,000. Patients with this disease visit various departments such as the department of internal medicine, otolaryngology, or dentistry. The symptoms of xerostomia can be alleviated by appropriate treatment with close cooperation between the dental and medical departments. In particular, dental treatment using direct approaches to the oral cavity is important. The effects of recovery of masticatory function on the salivary flow rate have been reported [2-4]. Therefore, we evaluated the possible improvement in xerostomia associated with systemic diseases after occlusal treatment.

\subsection{Relationship between Xerostomia and Drugs for Underlying Diseases}

There are many drugs that induce xerostomia. The drugs frequently inducing this disease are: 1) Drugs acting on the central and peripheral nerves and their receptors and 2) Drugs involved in electrolyte or water transfer [6].

Psychotropic drugs classified as category 1) are often used for a long period, and often induce inhibition of salivary secretion. Concerning the mechanism of this inhibition, some studies have suggested binding of these drugs to sympathetic and parasympathetic receptors on salivary gland cells, resulting in inhibition [1,6-9], These drugs not only alleviate tension in the central nervous system, reducing the instruction to secrete saliva but also inhibit the function of receptors on salivary gland cells and that of cell organelles. 
Table 4. Oral findings in Group 1.

\begin{tabular}{ccccccc}
\hline & $0 \mathrm{w}$ & $1-2 \mathrm{w}$ & Prosthesis fitting & $6 \mathrm{M}$ & $1 \mathrm{y}$ & $3 \mathrm{y}$ \\
\hline Findings & disease $\%$ & disease $\%$ & disease $\%$ & disease $\%$ & disease $\%$ & disease $\%$ \\
\hline Tongue redness & 75 & 40 & 5 & 5 & 0 & 0 \\
Tongue erosion & 65 & 20 & 5 & 0 & 0 & 0 \\
Tongue smoothness & 30 & 15 & 5 & 0 & 0 & 0 \\
Tongue fur & 50 & 25 & 5 & 0 & 0 & 0 \\
Palatal redness & 10 & 5 & 0 & 0 & 0 & 0 \\
Palatal erosion & 5 & 5 & 0 & 0 & 0 & 0 \\
Buccal mucosal redness & 35 & 20 & 0 & 0 & 0 & 0 \\
Buccal mucosal erosion & 5 & 10 & 0 & 0 & 0 & 0 \\
cheilosis angularis (erosion) & 15 & 10 & 0 & 0 & 0 & 0 \\
Oral aphtha & 5 & 5 & 0 & 0 & 0 \\
Oral ulcer & & 0 & 0 & 0 & 0 \\
\hline
\end{tabular}

Table 5. Oral findings in Group 2.

\begin{tabular}{|c|c|c|c|c|c|c|}
\hline & $0 \mathrm{w}$ & $1-2 w$ & Prosthesis fitting & $6 \mathrm{M}$ & $1 \mathrm{y}$ & $3 y$ \\
\hline Findings & disease $\%$ & disease $\%$ & disease $\%$ & disease $\%$ & disease $\%$ & disease $\%$ \\
\hline Tongue redness & 90 & 55 & 45 & 35 & 30 & 25 \\
\hline Tongue erosion & 85 & 50 & 35 & 15 & 10 & 15 \\
\hline Tongue smoothness & 60 & 45 & 25 & 20 & 20 & 20 \\
\hline Tongue fur & 70 & 40 & 25 & 10 & 0 & 0 \\
\hline Palatal redness & 35 & 25 & 20 & 15 & 15 & 5 \\
\hline Palatal erosion & 30 & 25 & 25 & 20 & 10 & 10 \\
\hline Buccal mucosal redness & 70 & 35 & 35 & 20 & 15 & 10 \\
\hline Buccal mucosal erosion & 20 & 20 & 20 & 10 & 5 & 10 \\
\hline cheilosis angularis (erosion) & 50 & 40 & 25 & 15 & 0 & 0 \\
\hline Oral aphtha & 35 & 30 & 25 & 15 & 5 & 0 \\
\hline Oral ulcer & 25 & 20 & 15 & 5 & 0 & 0 \\
\hline
\end{tabular}

One of the subjects in this study was receiving a psychotropic drug (second generation tricyclic antidepressant). This type of drug inhibits binding of acetylcholine to muscarinic receptors, inhibiting salivary secretion. However, prosthetic treatment slightly increased the resting salivary flow rate by $13 \%$ and the stimulated salivary flow rate by $4 \%$.

Drugs classified as category 2) include diuretics, which are subclassified according to the mechanism of action into osmotic and loop diuretics. As osmotic diuretics, two types that poorly permeate the cell membrane are often used. Disaccharides are negligibly reabsorbed by epithelial cells during their transfer through renal tubules, and excreted into the pelvis [10]. Since the salivary glands have no filtration system, when the blood osmolarity is high, water transfer from the blood vessels becomes difficult, causing difficulty in saliva production. Loop diuretics inhibit the action of ion transporters [10], and therefore, water returning to the blood decreases, resulting in an increase in the urine volume. In the salivary glands, since ion transporters are present on the blood side of the cell membrane of acinar cells, ion inflow into the cells is inhibited due to the effects of these drugs, and water movement becomes slow, resulting in a decrease in saliva secretion [11]. Diuretics are frequently used as anti-hypertensive drugs. In this study, 6 patients were receiving diuretics, but showed a $6 \%$ increase in the resting salivary flow rate and $60 \%$ increase in the stimulated salivary flow rate after prosthetic treatment.

As causes of a decreased salivary flow rate, some studies have suggested the influences of age and sex while others have suggested no influences of them. However, these studies have also shown the influences of drugs [6-11].

At the time of the initial consultation, Group 2 with underlying diseases showed a mean resting salivary flow 
rate $(\geq 1.73 \pm 0.43 \mathrm{ml} / 15 \mathrm{~min})$ above the reference value, but a mean stimulated salivary flow rate $(\geq 8.19 \pm 0.35$ $\mathrm{ml} / 10 \mathrm{~min}$ ) below the reference value. However, the mean stimulated salivary flow rate was above the reference values at the time of prosthesis fitting, and after 6 months, 1 year, and 3 months.

The incidence of drug-induced xerostomia differs among drugs, and is also affected by the number of drugs and drug administration period [12]. In this study, there were 3 patients with diabetic mellitus complicated by hypertension. The resting salivary flow rate at the first consultation in these patients was $\geq 2.16 \mathrm{ml} / 15 \mathrm{~min}$, being higher than the mean in Group 1 ( $\geq 1.73 \mathrm{ml} / 15 \mathrm{~min})$. The stimulated salivary flow rate in these patients was $\geq 8.66$ $\mathrm{ml} / 10 \mathrm{~min}$, being higher than the mean in Group $1(\geq 8.52$ $\mathrm{ml} / 15 \mathrm{~min})$, but below the reference value $(\geq 10 \mathrm{ml} /$ $15 \mathrm{~min})$. These results suggested certain influences of drugs and differences in the drug dose and administration period.

\subsection{Relationship between Underlying Diseases and the Salivary Flow Rate}

As diseases causing xerostomia, diabetes mellitus and Sjögren syndrome are particularly important. The incidence of diabetes mellitus including the borderline type is $10 \%$ of the Japanese population [12]. Sandberg et al. [13] showed a significantly high incidence of xerostomia in patients with diabetes mellitus. In general, dehydration due to osmotic diuretics induces xerostomia, but a decrease in saliva secretion itself has often been reported [14]. Association with decreased oral self-cleaning action, intractable changes of dental infections, or cheilosis angularis has also been suggested [15-17]. Brenardi et al. [15] reported a decrease in the salivary flow rate in patients with diabetes mellitus irrespective of control of the blood glucose level. Chavez et al. [16]. reported a significantly lower stimulated parotid salivary flow rate in elderly diabetic patients showing poor glycemic control ( $\mathrm{HbAlC}$ $>9 \%$ ) than in those showing good glycemic control or healthy subjects. Kao [17] performed salivary gland scintigraphy, and reported decreased salivary production and secretion in patients with type 2 diabetes mellitus complicated by xerostomia even in the presence good glycemic control, suggesting impairment of slivery gland function itself.

In this study, the resting salivary flow rate in the group with diabetes mellitus was $\geq 1.61 \mathrm{ml} / 10 \mathrm{~min}$, being above the reference value, and tended to gradually increase at the time of prosthetic fitting and after 1 and 3 years. In this group, the stimulated salivary flow rate was $\geq 8.39$ $\mathrm{ml} / 15 \mathrm{~min}$, being below the reference value, but also tended to increase at the time of prosthesis fitting and after 1 and 3 years.
Shögren syndrome is an organ-specific autoimmune disease [2-4], and the estimated number of patients with this disease is about 500,000. The symptoms include dry eyes, dry mouth, and dry nasal cavities. In the oral field, this disease damages the salivary glands, inducing salivary secretion disorder. Therefore, improvement in the salivary flow rate has reported to be difficult by improving masticatory function and the oral hygiene status [4].

Concerning changes in the salivary flow rate in the group with Sjögren syndrome, the resting salivary flow rate was $\geq 0.95 \mathrm{ml} / 10 \mathrm{~min}$ at the initial consultation, being below the reference value. In 1 patient, it increased to $1.60 \mathrm{ml} / 10 \mathrm{~min}$ after 6 months, exceeding the reference value, and further to $\geq 1.70 \mathrm{ml} / 10 \mathrm{~min}$ after 3 years. The other 3 patients showed a mean value of $\geq 0.90 \mathrm{ml} / 10 \mathrm{~min}$ after 6 months and $\geq 0.96 \mathrm{ml} / 10 \mathrm{~min}$ even after 3 years, being below the reference limit. The stimulated salivary flow rate was also below the reference value at the initial consultation. One patient showed an increase to $\geq 13.7$ $\mathrm{ml} / 10 \mathrm{~min}$ after $1-2$ weeks, exceeding the reference value, 2 showed an increase to $\geq 10.3 \mathrm{ml} / 10 \mathrm{~min}$ and $\geq 10.6 \mathrm{ml} / 10 \mathrm{~min}$, respectively, exceeding the reference value after 6 months, but the other showed $\geq 6.3 \mathrm{ml} /$ $10 \mathrm{~min}$, not exceeding the reference value $(\geq 10.0 \mathrm{ml} /$ $10 \mathrm{ml}$ ), even after 3 years. This patient had angina pectoris complicated by diabetes mellitus and was 79 years old. Since the drug administration period was long, recovery of the salivary flow rate was difficult by prosthetic treatment alone.

In the group with Sjögren syndrome, 2 patients had upper and lower complete dentures as the prosthetic status at the initial consultation, and the other 2 had upper and lower partial dentures. The treatment content was new upper and lower denture fabrication in 2 patients, new upper and lower partial denture fabrication in 1 , and adjustment of upper and lower partial dentures in the other. Since new dentures were fabricated in 3 patients, masticatory function may have slightly improved. These results suggest that the salivary flow rate can be improved by prosthetic treatment even in patients with Sjögren syndrome.

\subsection{Relationship between the Salivary Flow Rate and Oral Findings}

In Group 1 without underlying diseases, tongue redness (75\%), tongue erosion $(65 \%)$, and tongue fur $(50 \%)$ were frequently observed. With progression of oral hygiene instruction and prosthetic treatment, oral findings disappeared, and only tongue redness $(5 \%)$ was present after 6 months. After 1 year, there was no symptom or sign.

In Group 1, the mean resting salivary flow rate was $\geq 2.38 \mathrm{ml} / 15 \mathrm{~min}$ at the initial consultation, exceeding the reference value. The mean stimulated salivary flow rate 
was $\geq 11.17 \mathrm{ml} / 10$ minutes, exceeding the reference value. In this group, 12 of the 20 patients in this group showed a stimulated salivary flow rate below the reference value at the initial consultation but a value exceeding the reference value at the time of prosthesis fitting, suggesting effective recovery of occlusal function. Although it was reported that gustatory stimulation by food more markedly affects the salivary flow rate than mechanical stimulation given to the periodontal membrane or mucosa [18], these results suggest that masticatory stimulation also can increase the salivary flow rate.

In Group 2 with underlying diseases, many oral findings were present at the initial consultation: tongue redness $(90 \%)$, tongue erosion $(85 \%)$, tongue fur $(70 \%)$, buccal mucosal redness (70\%), tongue smoothness (60\%), cheilosis angularis (erosion) (50\%). At the time of prosthesis fitting, many oral findings were still observed: tongue redness $(45 \%)$, tongue erosion (35\%), tongue fur $(25 \%)$, buccal mucosal redness $(35 \%)$, tongue smoothness $(25 \%)$, cheilosis angularis (erosion) $(25 \%)$, palatal erosion $(25 \%)$, and oral aphtha $(25 \%)$.

Only the group with Sjögren syndrome showed tongue redness, tongue smoothness, palatal redness, and buccal mucosal redness after 1 year and 3 years. In this group, oral findings after 1 year and 3 years were present in $20 \%$, and tongue redness in $35 \%$ after 6 months, $30 \%$ after 1 year, and $25 \%$ after 3 years.

In Sjögren syndrome, lymphocyte invasion around the salivary gland ducts and disappearance of acini were reported [18]. Since the salivary glands themselves are damaged, mastication as mechanical stimulation may not induce changes in the salivary flow rate. In Group 2 with underlying disease, patients without improvement in oral findings tended to show only slight increases in both the resting and stimulated salivary flow rates.

The actions of saliva in the oral cavity are mucosal protection and repair. A decreased salivary flow rate reduces these actions, inducing aggravation of oral findings. A negative relationship between the salivary flow rate and Candida count has been reported [19-22]. Candida albicans, the main pathogen inducing oral candidiasis, is a member of the indigenous flora in $40 \%-50 \%$ of healthy people, but becomes pathogenic when the host immunity and infection defense function decreased. The provoking systemic causes include HIV infection, diabetes mellitus, radiotherapy or chemotherapy for malignant tumors, and long-term use of steroids and antibiotics. The local causes include wearing of ill-fitting dentures and decreased salivary sectretion [19].

Therefore, improvement in dentures that can be made by dentists can increase salivary secretion, which may contribute improvement in oral symptoms of xerostomia. One of treatment methods for xerostomia is elimination of inducing factors. Changes in drugs for systemic diseases and a reduction in the drug dose are difficult be- cause cooperation with medical institutions is necessary. However, if salivary secretion can be increased by dental approaches, improvement in xerostomia may be possible.

Based on the results of this study, complete cure of all cases of xerostomia associated with underlying diseases may be difficult by only increasing the salivary flow rate. Salivary secretion is adjusted by sympathetic and parasympathetic nerves. The activity of these nervous systems induces salivary secretion; a high activity of the parasympathetic nervous system induces more salivary secretion. Occlusal movements activating masticatory muscles reflexively stimulate parasympathetic nerves, resulting in salivary secretion. This suggests that recovery of occlusion after prosthetic treatment for the crown and missing teeth results in more active stimulation of parasympathetic nerves. Hoshi et al. reported improvement in indefinite complaints associated with xerostomia after recovery of occlusal function by prosthetic treatment and oral hygiene instruction [4,5]. In some cases, symptoms of xerostomia may improve after prosthetic treatment without special treatment methods such as drug administration.

We performed this study, speculating that an increase in salivary secretion can improve oral symptoms of xerostomia associated with systemic diseases. As a result, there were correlations among an increase in the salivary flow rate, improvement in oral findings, and improvement in oral dryness. However, in patients with Sjögren syndrome showing direct damage in the salivary gland tissue, since neither an increase in salivary secretion nor improvement in oral dryness was observed, selection of other treatment methods may be necessary. Thus, appropriate diagnosis of xerostomia including its cause is necessary in patients with systemic diseases. Based on this diagnosis, improvement may be achieved by dental treatment.

\section{CONCLUSION}

To improve symptoms of xerostomia associated with systemic diseases, we restored occlusal function to increase the salivary flow rate, and evaluated changes in oral findings and salivary secretion. As a result, improvement in occlusion led to an increase in the salivary flow rate and improvement in oral signs and symptoms. Thus, oral hygiene instruction and improvement in occlusion are useful for improving xerostomia, and can be used for treating this disease. In addition, their combination with other conventional treatment methods may produce more marked treatment effects.

\section{REFERENCES}

[1] Saito I. (2010) Dry mouth as a systemic disease. Progress in Medicine, 11, 2853-2857. 
[2] Hoshi, N., Mori, H., and Kimoto, K. (2011) Management of oral candidiasis in denture wearers. Journal of Prosthodontic Research, 55, 48-52. doi:10.1016/j.jpor.2010.03.004

[3] Hoshi, N. (2009) An attempt to recover mastication in a patient having a strong vomiting reflex. Japanese Journal of Oral Diagnosis/Oral Medicine, 22, 303-306.

[4] Mori, H., Hoshi, N. and Kimoto, K. (2011) Oral hygiene instruction and prosthetic treatment is related to improvement of salivary flow and oral symptoms. Japanese Journal of Oral Diagnosis/Oral Medicine, 24, 283-290.

[5] James, G. and Moore, P.A. (2003) Xerostomia: Etiology, recognition and treatment. The Journal of the American Dental Association, 1234, 61-69.

[6] Shigeo, Y. (2010) Infiuence of hyposalivation on oral Candida colonization. Oral Therapy of Pharmacology, 29, 19.

[7] Cerchiari, D.P., De Moricz, R.D., Sanjar F.A., et al. (2006) Burning mouth syndrome: Etiology. Brazilian Journal of Otorhinolaryngology, 72, 419-423.

[8] Heintze, U., Birkhed, D. and Bjorn, H. (1983) Secretion rate and buffer effect of resting and stiniulated whole saliva as function of age and sex. Swedish Dental Journal, 7, 227-238.

[9] Ntirhi, T.O., Meuman, J.H., Ainamo, A., et al. (1992) Association between salivary flow rate and the use of systemic medication among 76-, 81-, and 86-year-old inhabitants in Helsinki. Journal of Dental Research, 71, 1875-1880. doi:10.1177/00220345920710120401

[10] Kawaguchi, M. and Yamagishi, H. (2006) Coupling of benzodiazepine and $\operatorname{GABA}(\mathrm{A})$ receptors in the salivary glands is a factor of drug-induced xerostomia. Drug Research, 11, 291-296.

[11] Ogawa, T., Takadaa, K., Satoa, Y. and Chibab, H. (2010) The influence of causes of hyposalivation on clinical outcome of nizatidine in patients with dry mouth. Asian Journal of Oral and Maxillofacial Surgery, 31, 399-405.

[12] Watanabe, M., Kawaguchi, M. and Ishikawa, Y. (2006) Salivary glands and diabetic stress. Nihon Yakurigaku Zasshi. Folia Pharmacologica Japonica, 127, 273-277.

[13] Sandberg, G.E., Sundberg, H.E. and Fjellstrom. C.A.
(2000) Type 2 diabetes and oral health. A comparison between diabetic and non-diabetic subjects. Diabetes Research and Clinical Practice, 50, 27-34. doi:10.1016/S0168-8227(00)00159-5

[14] WADA, T. (2009) Two diabetic patients diagnosed through oral manifestati. Medical Society, 60, 22-25.

[15] Bernardi, M.J., Reis, A. and Loguercio, A.D. (2007) Study of the buffering capacity, $\mathrm{pH}$ and salivary flow rate in type 2 well-controlled and poorly controlled diabetic patients. Oral Health and Preventive Dentistry, 5, 73-78.

[16] Chabez, E.M., Taylor, G.W. and Borrell, L.N. (2000) Salivary function and glycemic control in older persons with diabetes. Oral Surgery, Oral Medicine, Oral Pathology, Oral Radiology and Endodontology, 89, 305-311. doi:10.1016/S1079-2104(00)70093-X

[17] Kao, C.H., Tsai, S.C. and Sun, S.S. (2001) Scintigraphic evidence of poor salivary function in type 2 diabetes. Diabetes Care, 24, 952-953. doi:10.2337/diacare.24.5.952-a

[18] Watanabe, S. and Dawes, C. (1988) A comparison of the effects of testing and chewing foods on the flow rate of whole saliva in man. Archives of Oral Biology, 33, 761764. doi:10.1016/0003-9969(88)90010-6

[19] Watanabe, A. (1995) Immunohistochemical study on irradiated human submandibular glands. Morphometrical Analysis of Histological Changes, Tsurumi University Dental Journal, 21, 247-265.

[20] Epstein, J.B., Pearsall, N.N. and Truelove, E.L. (1980) Quantitative relationships between Candida albicans in saliva and the clinical status of human subjects. Journal of Clinical Microbiology, 12, 475-476.

[21] Navazesh, M. (1995) Relationship between salivary flow rates and Candida albicans counts. Oral Surgery, Oral Medicine, Oral Pathology, Oral Radiology and Endodontology, 80, 284-288. doi:10.1016/S1079-2104(05)80384-1

[22] Torres, S.R., Peixoto, C.B., Caldas, D.M., et al. (2002) Relationship between salivary flow rates and Candida counts in subjects with xerostomia. Oral Surgery, Oral Medicine, Oral Pathology, Oral Radiology and Endodontology, 93, 149-154. doi:10.1067/moe.2002.119738 